\title{
Lipschitz Character of Solutions to the Inner Obstacle Problems
}

\author{
SŁAWOMIR JAGODZIŃSKI, ANNA OLEK AND KUBA SZCZEPANIAK
}

\begin{abstract}
In our paper we consider the inner problem with $l \in \mathbb{N}$ impediments from below, the inner problem with $m \in$ $\mathbb{N}$ impediments from above and the double inner problem with $l+m$ impediments. Assuming the Lipschitz character of the obstacles we show that the corresponding solutions are also Lipschitz. We extend here the result given in [SV], where the author considered the inner obstacle problem with a single impediment from below. Our work is based on the ideas introduced by J. Jordanov from 1982 who investigated $H^{1, p}(\Omega)$ regularity of solutions to inner obstacle problems.
\end{abstract}

\section{INTRODUCTION}

In many physical processes "obstacles" appear in a natural way having strong influence on the character of the examined problem. A simple example of such a situation is the study of contrast between a vibrating membrane and a vibrating membrane set between obstacles.

In the 1970's there was considerable interest in the analysis of obstacle problems. This was connected with the development of research on variational inequalities and has been studied by many authors (see $[\mathrm{BC}],[\mathrm{BS}],[\mathrm{T}]$ and references therein). The majority of results concentrated on, natural from a mathematical point of view, problems of existence and uniqueness of the solutions. However, in case of variational inequalities corresponding to obstacle problems additional questions regarding, e.g., the coincidence set (cf. [DS1], [DS2]) or regularity of the solutions (cf. [BS]) can be posed. These problems seem to be interesting due to possible applications.

2000 Mathematics Subject Classification. 35J85, 49J40.

Key words and phrases. Inner obstacle problems, Lipschitz continuity, variational inequalities. 
The fundamental result where regularity of solutions with regard to regularity of obstacles is studied in case of global obstacle problems can be found in $[\mathrm{KS}]$.

Recently the interest in the analysis of the obstacle problems has increased. This is due to appearance of works on the inner problems (see $[\mathrm{BSz}],[\mathrm{JOS}],[\mathrm{Ro}]$ and references therein). Among other things examination of regularity of solutions to the inner obstacle problems is a matter of significant importance.

A study of the Lipschitz character of the solutions to the obstacle problems was initiated in [SV]. The authors showed that the solution of the global problem and the inner problem with one obstacle from below is Lipschitz continuous assuming that the impediments are Lipschitz. Later on the papers [Ch1], [Ch2] appeared where one can find theorems concerning the Lipschitz continuity of the solutions to the global inverse and double global problems.

In our work we aim at transferring results concerning Lipschitz character of solutions of global obstacle problems to the case of inner ones. It is worth mentioning that the construction presented in our paper enables us to identify each inner problem with the corresponding global one. This fact makes it possible to carry out the complete analysis of the inner problems with the help of methods available for the global ones.

We offer a comprehensive study of the Lipschitz regularity of solutions of the inner obstacle problems. The present paper is a part of the research program on free boundary problems.

\section{Notation And Basic Definitions}

Throughout the paper we assume that $\Omega \subset \mathbb{R}^{n}$ is an open, bounded set with the smooth boundary $\partial \Omega$. The functions $a_{i j}: \bar{\Omega} \rightarrow \mathbb{R}$ for $1 \leq i, j \leq n$ belong to $C^{1}(\Omega)$ and satisfy the ellipticity condition, i.e., there exist $\gamma, \mu>0$ such that

$$
\mu|\xi|^{2} \geq a_{i j}(x) \xi_{i} \xi_{j} \geq \gamma|\xi|^{2} \quad \text { for } x \in \Omega \text { and } \xi \in \mathbb{R}^{n},
$$

where the summation convention is adopted. We also introduce the second order elliptic operator

$$
L=-\partial_{x_{i}}\left(a_{i j}(x) \partial_{x_{j}}\right) .
$$

Remark 2.1. The operator $L$ defined by (2) considered as the mapping $L: H_{0}^{1}(\Omega) \rightarrow H^{-1}(\Omega)$ defines (see $[\mathrm{KS}]$ ) a bilinear, continuous 
and coercive form on $H_{0}^{1}(\Omega)$ as follows:

$$
a(u, v)=\langle L u, v\rangle=\int_{\Omega} a_{i j}(x) u_{x_{i}}(x) v_{x_{j}}(x) d x \quad\left(u, v \in H_{0}^{1}(\Omega)\right) .
$$

Now we pass to the precise definitions of fundamental concepts of this work. Let us consider $l \in \mathbb{N}$ functions $\Psi_{i} \in H^{1}\left(E_{i}\right)$ where $E_{i} \subset \Omega$ are compact sets such that $\partial E_{i}$ is smooth, $E_{i} \cap E_{j}=\emptyset$ for $i, j=1, \ldots, l$ and $i \neq j$. Next we take $m \in \mathbb{N}$ functions $\Phi_{i} \in H^{1}\left(F_{i}\right)$ where $F_{i} \subset \Omega$ are compact sets such that $\partial F_{i}$ is smooth, $F_{i} \cap F_{j}=\emptyset$ for $i, j=1, \ldots, m$ and $i \neq j$. Moreover, we assume that:

$$
\Psi_{i} \leq \Phi_{j} \quad \text { on } E_{i} \cap F_{j} \text { for } i=1, \ldots, l \text { and } j=1, \ldots, m .
$$

We denote by $K_{l}, K^{m}$ and $K_{l}^{m}$ the following admissible sets:

$$
\begin{aligned}
K_{l}=\left\{v \in H_{0}^{1}(\Omega):\right. & \left.: v \geq \Psi_{i} \text { on } E_{i} \text { for } 1 \leq i \leq l\right\} \\
K^{m}= & \left\{v \in H_{0}^{1}(\Omega): v \leq \Phi_{i} \text { on } F_{i} \text { for } 1 \leq i \leq m\right\} \\
K_{l}^{m}= & \left\{v \in H_{0}^{1}(\Omega): v \geq \Psi_{i} \text { on } E_{i} \wedge v \leq \Phi_{j} \text { on } F_{j}\right. \\
& \text { for } 1 \leq i \leq l, 1 \leq j \leq m\}
\end{aligned}
$$

Definition 2.2. Let $l$ be a fixed natural number. For the form defined by (3) and $f \in H^{-1}(\Omega)$ the problem:

Find $u_{l} \in K_{l}$ such that

$$
a\left(u_{l}, v-u_{l}\right) \geq\left\langle f, v-u_{l}\right\rangle \quad \text { for any } v \in K_{l},
$$

where $K_{l}$ is defined by (5) is called an $l$-inner obstacle problem with the impediments $\Psi_{i}(i=1, \ldots, l)$.

We shall use the notation $l-I P$ to denote the $l$-inner obstacle problem.

Definition 2.3. Let $m$ be a fixed natural number. For the form defined by (3) and $f \in H^{-1}(\Omega)$ the problem:

Find $u^{m} \in K^{m}$ such that

$$
a\left(u^{m}, v-u^{m}\right) \geq\left\langle f, v-u^{m}\right\rangle \quad \text { for any } v \in K^{m},
$$

where $K^{m}$ is defined by (6) is called an $m$-inner inverse obstacle problem with the impediments $\Phi_{i}(i=1, \ldots, m)$.

We shall use the notation $m-I I P$ to denote the $m$-inner inverse obstacle problem.

Definition 2.4. Let $l, m$ be fixed natural numbers. For the form defined by (3) and $f \in H^{-1}(\Omega)$ the problem: 
Find $u_{l}^{m} \in K_{l}^{m}$ such that

$$
a\left(u_{l}^{m}, v-u_{l}^{m}\right) \geq\left\langle f, v-u_{l}^{m}\right\rangle \quad \text { for any } v \in K_{l}^{m},
$$

where $K_{l}^{m}$ is defined by (7) is called an $l, m$-double inner obstacle problem with the impediments $\Psi_{i}(i=1, \ldots, l)$ and $\Phi_{j}(j=$ $1, \ldots, m)$.

We shall use the notation $l, m-D I P$ to denote the $l, m$-double inner obstacle problem.

Remark 2.5. If we put $l=1$, take $E_{1}=\Omega$ and assume that $\Psi_{1}=\Psi$ satisfies $\left.\Psi\right|_{\partial \Omega} \leq 0$ then Definition 2.2 in fact is identical with the definition of the global obstacle problem with the impediment $\Psi$. The admissible set $\tilde{K}_{1}$ will be defined by

$$
\tilde{K}_{1}=\left\{v \in H_{0}^{1}(\Omega): v \geq \Psi \text { on } \Omega\right\} .
$$

We shall use the notation $G P$ to denote the global obstacle problem.

Remark 2.6. If we put $m=1$, take $F_{1}=\Omega$ and assume that $\Phi_{1}=\Phi$ satisfies $\left.\Phi\right|_{\partial \Omega} \geq 0$ then Definition 2.3 gives the definition of the inverse global obstacle problem with the impediment $\Phi$. The admissible set $\tilde{K}^{1}$ will be defined by

$$
\tilde{K}^{1}=\left\{v \in H_{0}^{1}(\Omega): v \leq \Phi_{i} \text { on } \Omega\right\} .
$$

We shall use the notation GIP to denote the inverse global obstacle problem.

Remark 2.7. If we put $l=m=1$, take $E_{1}=F_{1}=\Omega$ and assume that $\Psi_{1}=\Psi$ and $\Phi_{1}=\Phi$ are such that $\left.\Psi\right|_{\partial \Omega} \leq 0,\left.\Phi\right|_{\partial \Omega} \geq 0$ and $\Psi \geq \Phi$ then Definition 2.4 gives the definition of the double global obstacle problem with the impediments $\Psi$ and $\Phi$. The admissible set $\tilde{K}_{1}^{1}$ will be defined by

$$
\tilde{K}_{1}^{1}=\left\{v \in H_{0}^{1}(\Omega): \Psi \leq v \leq \Phi \text { on } \Omega\right\} .
$$

We shall use the notation $D G P$ to denote the double global obstacle problem.

The existence and uniqueness theorems for $G P, G I P, D G P$ can be found in $[\mathrm{KS}]$. 


\section{LipsChitz REgulaRity}

In this section we present the main results of our paper, i.e., the Lipschitz regularity of the solutions in the case of the inner obstacle problems. Since our approach is based on identification of the inner problem with the corresponding global one, we now recall the following lemma (see [SV], [Ch1], [Ch2]).

Lemma 3.1. The solutions: $\tilde{u}_{1}$ - of GP with the impediment $\Psi$ and $f=0, \tilde{u}^{1}$ - of GIP with the impediment $\Phi$ and $f=0, \tilde{u}_{1}^{1}$ of DGP with the impediments $\Psi, \Phi$ and $f=0$ are Lipschitz $\left(\tilde{u}_{1}, \tilde{u}^{1}\right.$, $\left.\tilde{u}_{1}^{1} \in H^{1, \infty}(\Omega)\right)$ provided $\Psi, \Phi \in H^{1, \infty}(\Omega)$.

We start with presenting the result for $l-I P$ (for $l \geq 1$ ). The following theorem is a generalisation of the one included in [SV] where $l=1$.

Theorem 3.2. If $\Psi_{i} \in H^{1, \infty}\left(E_{i}\right)$ then there exists a unique solution $u_{l}$ to $l-I P$ with the impediments $\Psi_{i}(i=1, \ldots, l)$. Moreover, if $f=0$ this solution is Lipschitz continuous.

Proof. Let us construct for each $i=1, \ldots, l$ the functions $\tilde{\Psi}_{i}: \Omega \rightarrow$ $\mathbb{R}$ in the following way

$$
\tilde{\Psi}_{i}= \begin{cases}w_{i} & \text { in } \Omega \backslash E_{i} \\ \Psi_{i} & \text { in } E_{i},\end{cases}
$$

where $w_{i} \in H^{1}\left(\Omega \backslash E_{i}\right)$ solves the following problem

$$
\begin{cases}L w_{i}=0 & \text { in } \Omega \backslash E_{i} \\ w_{i}=\Psi_{i} & \text { in } \partial E_{i} \\ w_{i}=0 & \text { in } \partial \Omega .\end{cases}
$$

Next we put

$$
\Psi=\max \left\{\tilde{\Psi}_{1}, \ldots, \tilde{\Psi}_{l}\right\}
$$

It is well known (see $[\mathrm{KS}]$ ) that in order to show existence and uniqueness of the solution $u_{l}$ of $l-I P$ it is enough to show non-emptiness of the set $K_{l}$. It is easy to see that $\Psi \in K_{l}$ which yields the desired conclusion.

On the other hand, we observe that $\tilde{\Psi}_{i}(i=1, \ldots, l)$ and consequently $\Psi$ are Lipschitz continuous. Then we notice that $u_{l}$ satisfies $L u_{l} \geq 0$ in $\Omega$. Indeed, for arbitrary $v \in H_{0}^{1}(\Omega)$ such that $v \geq 0$ in $\Omega$ we have $u_{l}+v \in K_{l}$. Thus we can write

$$
a\left(u_{l}, u_{l}+v-u_{l}\right) \geq 0 .
$$


The above inequality gives that $a\left(u_{l}, v\right) \geq 0$ for arbitrary $v \in H_{0}^{1}(\Omega)$ such that $v \geq 0$ in $\Omega$.

We have $\tilde{\tilde{\Psi}}_{i}=\Psi_{i}$ in $E_{i}, L \tilde{\Psi}_{i}=0$ in $\Omega \backslash E_{i},\left.\tilde{\Psi}_{i}\right|_{\partial \Omega}=0$ and $L u_{l} \geq 0$ in $\Omega \backslash E_{i}$. Therefore using the maximum principle we derive that $u_{l} \geq \tilde{\Psi}_{i}$ in $\Omega$.

Moreover, we have that $u_{l} \geq \Psi$ in $\Omega$. It is true due to the fact that $u_{l} \geq \tilde{\Psi}_{i}$ in $\Omega$ for $i=1, \ldots, l$. This together with $u_{l} \in H_{0}^{1}(\Omega)$ implies that $u_{l} \in \tilde{K}_{1}$ where $\tilde{K}_{1}$ is defined in (11).

Let us denote by $\tilde{u}_{1}$ the solution to $G P$ with the impediment $\Psi$ and $f$ being equal to zero. Since $u_{l} \in \tilde{K}_{1}$ we can state that

$$
a\left(\tilde{u}_{1}, u_{l}-\tilde{u}_{1}\right) \geq 0 .
$$

On the other hand, $\tilde{K}_{1} \subset K_{l}$ since if $v \in \tilde{K}_{1}$ then $v \in H_{0}^{1}(\Omega)$ and $v \geq \Psi \geq \Psi_{i}$ on $E_{i}$ for $i=1, \ldots, l$. Hence we can write

$$
a\left(u_{l}, \tilde{u}_{1}-u_{l}\right) \geq 0,
$$

because $u_{l} \in K_{l}$ solves the variational inequality (8) with $f=0$. Having added the last two inequalities we shall obtain (using the coercivity of the form $a(\cdot, \cdot)$ in $\left.H_{0}^{1}(\Omega)\right)$ that there exists $\nu>0$ such that

$$
\nu\left\|\tilde{u}_{1}-u_{l}\right\|^{2} \leq a\left(\tilde{u}_{1}-u_{l}, \tilde{u}_{1}-u_{l}\right) \leq 0,
$$

which implies that $\tilde{u}_{1}=u_{l}$ in $\Omega$. Lipschitz continuity of $\tilde{u}_{1}$ (see Lemma 3.1) completes the proof.

The result similar to the one presented in Theorem 3.2 can be obtained for $m-I I P(m \geq 1)$.

Theorem 3.3. If $\Phi_{i} \in H^{1, \infty}\left(F_{i}\right)$ then there exists a unique solution $u^{m}$ to $m-I I P$ with the impediments $\Phi_{i}(i=1, \ldots, m)$. Moreover, if $f=0$ this solution is Lipschitz continuous.

The proof of Theorem 3.3 is almost identical to the previous one, so we omit it.

In the third theorem we consider $1,1-D I P$. In this case we extend the result given in [SV] by adding one impediments from above.

Theorem 3.4. If the functions $\Psi_{1} \in H^{1, \infty}\left(E_{1}\right), \Phi_{1} \in H^{1, \infty}\left(F_{1}\right)$ satisfy

$$
\Psi_{1} \leq \Phi_{1} \quad \text { in } E_{1} \cap F_{1},
$$


then there exists a unique solution $u_{1}^{1}$ to $1,1-D I P$ with the impediments $\Psi_{1}, \Phi_{1}$. Moreover, if $f=0$ this solution is Lipschitz continuous.

Proof. Firstly, applying the ideas presented in the proof of Theorem 3.2 (see (14)) we construct the Lipschitz extensions of $\Psi_{1}$ and $\Phi_{1}$ onto the whole domain $\Omega$. In order to reduce the complexity of notations those extensions we still call $\Psi_{1}$ and $\Phi_{1}$. Then we observe that $\max \left\{\Psi_{1}, 0\right\}+\min \left\{\Phi_{1}, 0\right\} \in K_{1}^{1}$ which gives us existence of the unique solution $u_{1}^{1} \in K_{1}^{1}$ to the $1,1-D I P$.

In the proof we shall construct two Lipschitz functions $\Psi$ and $\Phi$ such that the solution $\tilde{u}_{1}^{1}$ of $D G P$ with the impediments $\Psi$ and $\Phi$ and $f$ being equal to zero will coincide with $u_{1}^{1}$ - the solution of $1,1-D I P$.

Let us consider the coincidence set $I\left[u_{1}^{1}\right]$ for $1,1-D I P$. Obviously it is contained in $E_{1} \cup F_{1}$ (as (16) holds). We denote by $I_{F}\left[u_{1}^{1}\right]$ that part of $I\left[u_{1}^{1}\right]$ which is contained in $F_{1} \cap \overline{\left(\Omega \backslash E_{1}\right)}$ where $u_{1}^{1}=\Phi_{1}$ and by $I_{E}\left[u_{1}^{1}\right]$ that part of $I\left[u_{1}^{1}\right]$ which is contained in $E_{1} \cap \overline{\left(\Omega \backslash F_{1}\right)}$ where $u_{1}^{1}=\Psi_{1}$. Now we define

$$
\begin{aligned}
& \tilde{\Psi}_{1}= \begin{cases}\Psi_{1} & \text { in } E_{1} \\
\min \left\{\Psi_{1}, \Phi_{1}\right\} & \text { in } \Omega \backslash E_{1},\end{cases} \\
& \tilde{\Phi}_{1}= \begin{cases}\Phi_{1} & \text { in } F_{1} \\
\max \left\{\Psi_{1}, \Phi_{1}\right\} & \text { in } \Omega \backslash F_{1} .\end{cases}
\end{aligned}
$$

Both functions $\tilde{\Psi}_{1}$ and $\tilde{\Phi}_{1}$ are continuous. Moreover, they are both Lipschitz.

Now let us take a Lipschitz function $\xi \in H_{0}^{1, \infty}(\Omega)$ such that $\left.\xi\right|_{\partial\left(\Omega \backslash E_{1}\right)}=0$ and $\xi<0$ in $\Omega \backslash E_{1}$. Next we consider the Lipschitz function $\delta \in H^{1, \infty}(\Omega)$ where we put $\delta=\tilde{\Psi}_{1}+\xi$. We know that

$$
u_{1}^{1}=\Phi_{1}=\tilde{\Phi}_{1} \quad \text { in } \mathrm{I}_{\mathrm{F}}\left[\mathrm{u}_{1}^{1}\right] .
$$

It also satisfies

$$
u_{1}^{1}>\delta=\tilde{\Psi}_{1}+\xi \quad \text { in } \mathrm{I}_{\mathrm{F}}\left[\mathrm{u}_{1}^{1}\right]
$$

since $\tilde{\Psi}_{1}+\xi=\min \left\{\Psi_{1}, \Phi_{1}\right\}+\xi<\tilde{\Phi}_{1}$ in $I_{F}\left[u_{1}^{1}\right]$. From the continuity of $u_{1}^{1}, \xi$ and $\tilde{\Psi}_{1}$ we state that there exists a neighbourhood $O_{F}$ of $I_{F}\left[u_{1}^{1}\right]$ where the inequality (20) holds.

Now we choose a set $D_{F}$ with the smooth boundary in the following way:

$$
I_{F}\left[u_{1}^{1}\right] \subset D_{F} \subset \bar{D}_{F} \subset O_{F} \cap \overline{\left(\Omega \backslash E_{1}\right)} .
$$


Let $\psi$ be the solution of the problem:

$$
\begin{cases}L \psi=0 & \text { in }\left(\Omega \backslash E_{1}\right) \backslash \bar{D}_{F} \\ \psi=\delta & \text { in } \partial\left(\left(\Omega \backslash E_{1}\right) \backslash \bar{D}_{F}\right) .\end{cases}
$$

The function $\psi$ is Lipschitz (see $[\mathrm{ADN}],[\mathrm{BC}]$ ). Next we remark that the set $D$ was chosen in such a way that $\left(\Omega \backslash E_{1}\right) \backslash \bar{D}_{F} \subset \Omega \backslash I\left[u_{1}^{1}\right]$. Therefore using again the basic properties of the solutions to the obstacle problems (see $[\mathrm{KS}]$ ) we have that $L u_{1}^{1}=0$ in $\left(\Omega \backslash E_{1}\right) \backslash \bar{D}_{F}$. Hence

$$
L\left(u_{1}^{1}-\psi\right)=0 \quad \text { in }\left(\Omega \backslash \mathrm{E}_{1}\right) \backslash \overline{\mathrm{D}}_{\mathrm{F}} .
$$

Moreover, we have

$$
u_{1}^{1}=\psi=\Psi_{1} \quad \text { in } \partial \Omega,
$$

which follows from the definition of extension of $\Psi_{1}$ and the constructions of $\tilde{\Psi}_{1}$ and $\xi$,

$$
u_{1}^{1} \geq \psi=\Psi_{1} \quad \text { in } \partial E_{1},
$$

which follows from (16) and the constructions of $\tilde{\Psi}_{1}$ and $\xi$,

$$
u_{1}^{1} \geq \psi \quad \text { in } \partial D_{F},
$$

which follows from $(20)$ and the construction of the set $D_{F}$. Then the maximum principle implies that

$$
u_{1}^{1} \geq \psi \quad \text { in }\left(\Omega \backslash \mathrm{E}_{1}\right) \backslash \overline{\mathrm{D}}_{\mathrm{F}} .
$$

Finally we put

$$
\Psi= \begin{cases}\Psi_{1} & \text { in } E_{1} \\ \delta & \text { in } \bar{D}_{F} \\ \psi & \text { in }\left(\Omega \backslash E_{1}\right) \backslash \bar{D}_{F} .\end{cases}
$$

Clearly the function $\Psi$ is Lipschitz continuous in $\Omega$. Moreover,

$$
u_{1}^{1} \geq \Psi \quad \text { in } \Omega
$$

since $u_{1}^{1} \in K_{1}^{1},(22)$ holds and (20) is satisfied in $D \subset O_{F} \cap \overline{\left(\Omega \backslash E_{1}\right)}$.

Now we pass to the remaining part of the proof. We choose a Lipschitz function $\eta \in H_{0}^{1, \infty}(\Omega)$ such that $\eta>0$ in $\Omega \backslash F_{1}$ and $\left.\eta\right|_{\partial\left(\Omega \backslash F_{1}\right)}=0$. Next we consider the Lipschitz function $\sigma \in H^{1, \infty}(\Omega)$ where we put $\sigma=\tilde{\Phi}_{1}+\eta$. We know that $u_{1}^{1}=\tilde{\Psi}_{1}=\Psi_{1}$ in $I_{E}\left[u_{1}^{1}\right]$. It also satisfies the following:

$$
u_{1}^{1}<\sigma=\tilde{\Phi}_{1}+\eta \quad \text { in } I_{E}\left[u_{1}^{1}\right]
$$


as $\tilde{\Psi}_{1}=\Psi<\tilde{\Phi}_{1}+\eta$ in $I_{E}\left[u_{1}^{1}\right]$. From the continuity of $u_{1}^{1}, \eta$ and $\tilde{\Phi}_{1}$ we state that there exists a neighbourhood $O_{E}$ of $I_{E}\left[u_{1}^{1}\right]$ where the inequality (25) holds. Acting similarly as above we can choose a set $D_{E}$ with smooth boundary such that:

$$
I_{E}\left[u_{1}^{1}\right] \subset D_{E} \subset \bar{D}_{E} \subset O_{E} \cap\left(\Omega \backslash F_{1}\right) .
$$

Denoting by $\phi$ the solution of the problem

$$
\begin{cases}L \phi=0 & \text { in }\left(\Omega \backslash F_{1}\right) \backslash \bar{D}_{E} \\ \phi=\sigma & \text { in } \partial\left(\left(\Omega \backslash F_{1}\right) \backslash \bar{D}_{E}\right),\end{cases}
$$

we get that $\phi$ is Lipschitz provided $\sigma$ is Lipschitz. Knowing that $\left(\Omega \backslash F_{1}\right) \backslash \bar{D}_{E} \subset \Omega \backslash I\left[u_{1}^{1}\right]$ and using the maximum principle we deduce that:

$$
u_{1}^{1} \leq \phi \quad \text { in }\left(\Omega \backslash \mathrm{F}_{1}\right) \backslash \overline{\mathrm{D}}_{\mathrm{E}} .
$$

Finally we put

$$
\Phi= \begin{cases}\Phi_{1} & \text { in } F_{1} \\ \sigma & \text { in } \bar{D}_{E} \\ \phi & \text { in }\left(\Omega \backslash F_{1}\right) \backslash \bar{D}_{E} .\end{cases}
$$

Clearly the function $\Phi$ is Lipschitz. Moreover,

$$
u_{1}^{1} \leq \Phi \quad \text { in } \Omega
$$

as $u_{1}^{1} \in K_{1}^{1}$, (27) holds and (25) is satisfied in $D_{E} \subset O_{E} \cap \overline{\Omega \backslash F_{1}}$.

Conditions (24), (29) together with $u_{1}^{1} \in H_{0}^{1}(\Omega)$ imply that $u_{1}^{1} \in$ $\tilde{K}_{1}^{1}$, where $\tilde{K}_{1}^{1}$ is defined in (12). Moreover, $\tilde{K}_{1}^{1} \subset K_{1}^{1}$. Indeed, if $v \in \tilde{K}_{1}^{1}$ then $v \in H_{0}^{1}(\Omega), v \geq \Psi=\Psi_{1}$ in $E_{1}$ and $v \leq \Phi=\Phi_{1}$ in $F_{1}$ which gives that $v \in K_{1}^{1}$.

Let us denote by $\tilde{u}_{1}^{1}$ the solution of the $D G P$ with the impediments $\Psi, \Phi$ given by (23), (28), respectively and $f$ being equal to zero. Using coercivity of the form $a(\cdot, \cdot)$ on $H_{0}^{1}(\Omega)$ we shall deduce that $u_{1}^{1}=\tilde{u}_{1}^{1}$. This completes the proof (as $\tilde{u}_{1}^{1}$ is Lipschitz - see Lemma 3.1).

The last theorem describes the Lipschitz continuity of the solutions in the most general case, i.e., $l, m-D I P$.

Theorem 3.5. If the functions $\Psi_{i} \in H^{1, \infty}\left(E_{i}\right)(i=1, \ldots, l), \Phi_{j} \in$ $H^{1, \infty}\left(F_{j}\right)(j=1, \ldots, m)$ satisfy

$$
\Psi_{i} \leq \Phi_{j} \quad \text { in } E_{i} \cap F_{j},
$$


then there exists a unique solution $u_{l}^{m}$ to $l, m-D I P$ with the impediments $\Psi_{i}, \Phi_{j}$. Moreover, if $f=0$ this solution is Lipschitz continuous.

Proof. At the beginning similarly to what we did in the proof of Theorem 3.2 (see (14)) we construct the Lipschitz extensions of $\Psi_{i}$ and $\Phi_{j}$ onto the whole domain $\Omega$. Again in order to avoid too much complexity these extensions will be still called $\Psi_{i}$ and $\Phi_{j}$. Then we observe that $\max \left\{\Psi_{1}, \ldots, \Psi_{l}, 0\right\}+\min \left\{\Phi_{1}, \ldots, \Phi_{m}, 0\right\} \in K_{l}^{m}$ which gives us existence of the unique solution $u_{l}^{m} \in K_{l}^{m}$ to $l, m-D I P$.

It could be proved (see $[\mathrm{ES}]$ ) that the following estimates for the solutions to the inner obstacle problems are satisfied

$$
u_{1(i)}^{m} \leq u_{l}^{m} \leq u_{l}^{1(j)} \quad \text { in } \Omega,
$$

where $u_{1(i)}^{m}$ denotes the solution of $1, m-D I P$ with an arbitrarily fixed impediment $\Psi_{i}$ and $m$ impediments $\Phi_{j}, u_{l}^{1(j)}$ is the solution of $l, 1-D I P$ with $l$ impediments $\Psi_{i}$ and an arbitrarily fixed $\Phi_{j}$.

Firstly, we examine 1, $m-D I P$ with the fixed impediment $\Psi_{i}$ and $m$ impediments $\Phi_{j}$. Using the construction presented in the proof of Theorem 3.4 for each pair of the obstacles $\left(\Psi_{i}, \Phi_{j}\right)$ we construct $m$ global obstacles $\tilde{\Phi}_{j}$ according to $(28)$ and next we put $\Phi^{i}=\min _{1 \leq j \leq m} \tilde{\Phi}_{j}$. Then we consider $1,1-D I P$ with the admissible set

$$
K_{1(i)}^{1}=\left\{v \in H_{0}^{1}(\Omega): v \geq \Psi_{i} \text { on } E_{i} \wedge v \leq \Phi^{i} \text { on } \Omega\right\} .
$$

Its solution exists and we denote it by $u_{1(i)}^{1}$.

It can be easily seen that $u_{1(i)}^{m} \in K_{1(i)}^{1}$ since (29) is satisfied for all $j=1, \ldots, m$. On the other hand, $K_{1(i)}^{1} \subset K_{1(i)}^{m}$. Indeed, if we take $v \in K_{1(i)}^{1}$ then $v \geq \Psi_{i}$ on $E_{i}$ and $v \leq \Phi^{i}=\min _{1 \leq j \leq m} \tilde{\Phi}_{j}$ and $\tilde{\Phi}_{j}=\Phi_{j}$ on $F_{j}$. Using the coerciveness of the form $a(\cdot, \cdot)$ on $H_{0}^{1}(\Omega)$ we get that $u_{1(i)}^{m}=u_{1(i)}^{1}$.

Having $1,1-D I P$ with the pair of obstacles $\left(\Psi_{i}, \Phi^{i}\right)$ we apply once again the construction described in the proof of the previous theorem and we create the function $\bar{\Psi}_{i}$ according to (23). Obviously

$$
u_{1(i)}^{m} \geq \bar{\Psi}_{i} \quad \text { on } \Omega \text {. }
$$

Then we consider $l, 1-D I P$ with the fixed impediment $\Phi_{j}$. For each pair of the obstacles $\left(\Psi_{i}, \Phi_{j}\right)$ we build $l$ global obstacles $\tilde{\Psi}_{i}$ according to $(23)$ and next we put $\Psi^{j}=\max _{1 \leq i \leq l} \tilde{\Psi}_{i}$. Then we consider 
1,1 - DIP with the admissible set

$$
K_{1}^{1(j)}=\left\{v \in H_{0}^{1}(\Omega): v \leq \Psi^{j} \text { on } \Omega \wedge v \leq \Phi_{j} \text { on } F_{j}\right\} .
$$

Its solution exists and we call it $u_{1}^{1(j)}$.

One can observe that $u_{l}^{1(j)} \in K_{1}^{1(j)}$ since (24) is satisfied for all $i=1, \ldots, l$. Moreover, $K_{1}^{1(j)} \subset K_{l}^{1(j)}$. Indeed, if we take $v \in K_{1}^{1(j)}$ then $v \leq \Phi_{j}$ on $F_{j}$ and $v \geq \Psi^{j}=\max _{1 \leq i \leq l} \tilde{\Psi}_{i}$ and $\tilde{\Psi}_{i}=\Psi_{i}$ on $E_{i}$. Using the coerciveness of the form $a(\cdot, \cdot)$ on $H_{0}^{1}(\Omega)$ we get that $u_{l}^{1(j)}=u_{1}^{1(j)}$.

Concentrating once again on 1,1-DIP with the pair of obstacles $\left(\Psi^{j}, \Phi_{j}\right)$ we create using $(28)$ the global impediment $\bar{\Phi}_{j}$ such that

$$
u_{l}^{1(j)} \leq \bar{\Phi}_{j} \quad \text { on } \Omega \text {. }
$$

At this moment we deal with two Lipschitz function $\bar{\Psi}_{i}, \bar{\Phi}_{j}$ satisfying (33), (35), respectively. Repeating for all $i=1, \ldots, l$ and for all $j=1, \ldots, m$ the constructions described above we can build $l$ functions $\bar{\Psi}_{i}(i=1, \ldots, l)$ and $m$ functions $\bar{\Phi}_{j}(j=1, \ldots, m)$. Then we define:

$$
\Psi=\max _{i=1, \ldots, l} \bar{\Psi}_{i}, \quad \Phi=\min _{j=1, \ldots, m} \bar{\Phi}_{j} .
$$

From (31) we get the following estimates which hold for arbitrary $i, j$

$$
\bar{\Psi}_{i} \leq u_{1(i)}^{m} \leq u_{l}^{m} \leq u_{l}^{1(j)} \leq \bar{\Phi}_{j} .
$$

Thus

$$
\Psi \leq u_{l}^{m} \leq \Phi .
$$

This together with $u_{l}^{m} \in H_{0}^{1}(\Omega)$ implies that $u_{l}^{m} \in \tilde{K}_{1}^{1}$, where $\tilde{K}_{1}^{1}$ is defined in (13). Moreover, due to definition of $\Psi$ and $\Phi$, it is easy to see that $\tilde{K}_{1}^{1} \subset K_{l}^{m}$.

Let us denote by $\tilde{u}_{1}^{1}$ the solution of $D G P$ with the impediments $\Psi$ and $\Phi$. Using the coercivity of the form $a(\cdot, \cdot)$ in $H_{0}^{1}(\Omega)$ we deduce that $u_{l}^{m}$ equals $\tilde{u}_{1}^{1}$ which is Lipschitz continuous (see Lemma 3.1). This completes the proof.

Remark 3.6. It is well known that in case of solutions of the global problems one can expect their regularity up to $H^{2, p}$. For the inner problem the situation is much more complicated. Despite $H^{2, p}$ regularity of the obstacle the same class of the solution can not be obtained. However under certain assumptions it is possible to get $H^{2, p}$ regularity of the solutions (see [BS], [JOS]). 
Remark 3.7. It is worth pointing out that it was possible to adopt the method of identification of the inner problem with the global one to obtain the results concerning continuous dependence on obstacle of solutions (see [OS], [JOS]).

\section{REFERENCES}

[ADN] S. Agmon, A. Douglis, L. Nirenberg, Estimates near the boundary for the solution of partial differential equations satisfying general boundary conditions, Comm. Pure Appl. Math. 12 (1959), 623-727.

[BC] C. Baiocchi, A. Capelo, Variational and quasivariational inequalities, J. Wiley and Sons, 1984.

[Br] H. Brezis, Problemes unilatreaux, J. Math. Pures Appl. 51 (1972), 1-168.

[BS] H. Brezis, G. Stampacchia, Sur la regularite de la solution d'inequations elliptiques, Bull. Soc. Math. France 96 (1968), 153-180.

[BSz] J. Baniasiak, K. Szczepaniak, On regularity of solutions to inner obstacle problems, Zeitschrift für Analysis und ihre Anwendungen 12 (1993), 401404.

[Ch1] M. Chipot, Sur la regularite Lipschitzienne de la solution d'inequations elliptiques, J. Math. Pures Appl. 57 (1978), 69-76.

[Ch2] M. Chipot, Variational inequlities and flow through the porous media, Springer Verlag, 1984.

[DS1] I. Dziubiński, J. Szczepaniak, Starshapedness of the level sets and coincidence set in the global obstacle problem with non-zero force, Bull. De la Soc. Des Sciences et des lettres de Lodz 31 (2000), 121-125.

[DS2] I. Dziubiński, J. Szczepaniak, Coincidence stes in The Special Case of the Dirichlet Obstacle Problems, Demonstratio Mathematica 27 (1994), 347-350.

[ES] T. Ekholm, J. Szczepaniak, Applications of Lions-Stampacchia's Theorem to the Obstacle Problems, K.T.H, 2000, 1-59.

[GT] D. Gilbarg, N. S. Trudinger, Elliptic partial differential equations of second order, Springer Verlag, 1977.

[Jo] J. Jordanov, Solutions holderiennes d'inequations variationelles a contraines discontinuons, Serdica 8 (1982), 296-306.

[JOS] S. Jagodziński, A. Olek, J. Szczepaniak, Inner Obstacle Problems: Continuous Dependence on Obstacle and Regularity of the Solutions, to appear.

[KS] D. Kinderlehrer, G. Stampacchia, An introduction to variational inequalities and their applications, Academic Press, 1980.

[OS] A. Olek, K. Szczepaniak, Continuous dependence on obstacle in double global obstacle problems, Annales Academiæ Scientiarum Fennicæ Mathematica 28 (2003), 89-97.

[Ro] J.F.Rodriquez, Obstacle Problems in Mathematical Physics, North Holland, 1987.

[SV] G. Stampacchia, A. Vignoli, A remark for a second order nonlinear differential operator with non-Lipschitz obstacles, Boll. Un. Mat. Ital. 5 (1972), 123-131. 
[T] G. M. Troianello, Elliptic differential equations and obstacle problems, Plenum Press, 1987.

Sławomir Jagodziński, Anna Olek and Kuba Szczepaniak, Department of Mathematics,

Technical University of Łódź,

Al. Politechniki 11,

90-924 Łódź, Poland

ao@sunlib.p.lodz.pl

Received on 9 September $200 \%$. 